\title{
Investigation of Chaotic and Strange Nonchaotic Phenomena in Nonautonomous Wien-Bridge Oscillator with Diode Nonlinearity
}

\author{
R. Rizwana and I. Raja Mohamed \\ Department of Physics, B. S. Abdur Rahman University, Chennai 600 048, India \\ Correspondence should be addressed to R. Rizwana; rizwana28282@yahoo.in
}

Received 30 September 2014; Revised 15 December 2014; Accepted 19 December 2014

Academic Editor: Thordur Runolfsson

Copyright (c) 2015 R. Rizwana and I. Raja Mohamed. This is an open access article distributed under the Creative Commons Attribution License, which permits unrestricted use, distribution, and reproduction in any medium, provided the original work is properly cited.

\begin{abstract}
We have studied the chaotic and strange nonchaotic phenomena of a simple quasiperiodically forced Wien bridge oscillator circuit with diode as the only nonlinearity in this electronic oscillator system responsible for various nonlinear behaviors. Both the experimental results and the numerical simulation results for their confirmation are provided to show the bifurcation process. Various measures used for the numerical confirmation of SNA are power spectrum, maximal Lyapunov exponent, path of translational variables, mean square displacement, projection of poincaré section, log-log plot, and autocorrelation function. Based upon the numerical results, the birth of SNAs has been identified in the band merging route, intermittency route, and blowout bifurcation route. In addition, the birth of SNAs has been analyzed with peculiar mechanism, namely, "0-1 Test" employing the one state dynamical variable.
\end{abstract}

\section{Introduction}

Following the theoretical study of Grebogi et al. [1] on SNAs, in the recent years enormous attention has been paid to study the dynamics of SNAs which makes a fractal walk in its complex plane, possessing both discrete and continuous components in its spectral plane [2-4]. As evidence, SNAs are geometric structures possessing characteristic properties existing in the transitional state between periodicity and aperiodic chaotic dynamics. They are often expected to be generic in nonlinear systems excited by at least two periodic forces possessing incommensurate (or) irrational frequencies. Hence, the nature of quasiperiodic force with irrational frequencies plays an essential part in the generation of SNAs. In particular, the SNAs have been realized in a varietal quasiperiodically perturbed physical situations such as in pendulums [5], quantum particles [6], biologically operated oscillators [7], Duffing-type oscillators [8], velocity based oscillators [9], and electronic system $[3,10]$ and in specific maps [11]. Also the practical observation of these quaint attractors is made possible in the following experiments, quasiperiodically forced system of buckled magneto elastic ribbon [12], in analog simulating model of multistable potential [13] and neon glow discharge experiment [14]. Furthermore, SNAs take part in the Anderson localization in the Schrodinger equation influenced with quasiperiodic potential [15]. Consequently, several routes and their mechanisms leading to the birth of SNAs have been described $[9,10]$. The common routes are (i) torus doubling sequence to SNA to chaos [16, 17], (ii) gradual fractalization of torus to SNA to chaos [18], (iii) blowout bifurcation route to SNA [19], (iv) type I intermittency route to SNA [20], (v) type III intermittency route to SNA [8], (vi) birth of SNAs due to reemerging torus doubling bifurcation [9]. The synchronization between SNAs paves an essential application in the field of secure communication, cryptography, and so forth [21-24].

In the present work, we consider standard Wien bridge oscillator which is a familiar electronic linear oscillator. It is driven by a quasiperiodic force and brought into the hierarchy of nonlinear state employing an ordinary component, diode as a nonlinear device. The circuit design and its 

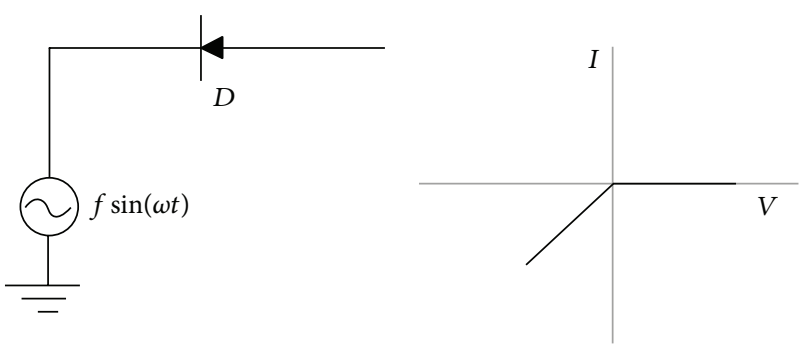

FIGURE 1: Diode and its piecewise-linear characteristic function (voltage $V$-current $I$ ).

implementation are described in Section 2. The experimental results obtained from the electronic system are verified with the numerical simulation of the state equations of the modified Wien bridge oscillator system (Section 3). The various dynamical states emerging in the system behavior are discussed in Section 3.1. The "0-1 test" is found useful to analyze the creation of SNA in the system (Section 3.2). The other numerical studies characterizing the property of SNA have been carried out (Section 3.3). The results are concluded in Section 4.

\section{Circuit Realization}

2.1. System Design. In this paper, the modified nonautonomous Wien bridge oscillator [25] is considered, with additional external sinusoidal force. The quasiperiodically forced system contains a standard Wien bridge oscillator with a diode, used as nonlinear element.

The characteristic function of a diode is a nonlinear piecewise segment (Figure 1). The voltage across the diode is related nonlinearly to its current through the relation, $I=I_{0}\left(e^{(q v / \eta k T)-1}\right)$. Hence, it is a nonlinear element that performs switching operation which gets shorted on forward bias with minimal resistance and opened on reverse bias with infinite resistance. Based on these basic properties, diodes can be implicated in oscillatory systems for the investigation of nonlinear phenomena.

The purpose of this design (Figure 2) is to explore the possible complex dynamics obtained from a nonlinear form of simple linear oscillator and to investigate the ensuing chaotic and strange nonchaotic signals, thereby verifying their states with the appropriate characterizing methods.

2.2. System Equations. The electronic system implemented consists of an operational amplifier (OP AMP $\mu$ A741), four resistors, two capacitors, and a diode (IN4148) and external sinusoidal periodic signals from two function generator (AGILENT 33220A). The chosen parameter fixed values are $R_{1}=R_{2}=3.3 \mathrm{~K}, R_{i}=1 \mathrm{~K}, R_{f}=3 \mathrm{~K}, C_{1}=C_{2}=68 \mathrm{nF}$. The state equations (1) of the designed system (Figure 2) so obtained by applying the Kirchoff's laws at the appropriate nodes of the circuit are required for dynamical analyses through numerical simulation. The governing normalized

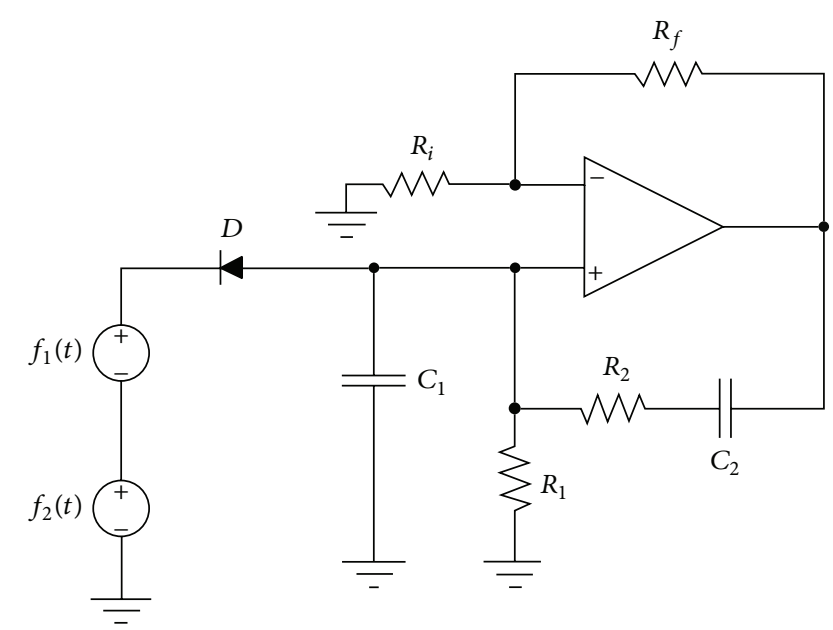

FIGURE 2: Experimental setup of quasiperiodically forced Wien bridge Oscillator.

equations of the dynamical system are in the form of first order differential equation:

$$
\begin{gathered}
\frac{d x_{1}}{d t}=(k-2) x_{1}-x_{2}-G(y), \\
\frac{d x_{2}}{d t}=(k-1) x_{1}-x_{2},
\end{gathered}
$$

where $G(y)=0.5 b(|y-1|+y-1)$ with $b=32$ and $y=$ $x_{1}-\left(f_{1} \sin \omega_{1} t+f_{2} \sin \omega_{2} t\right)$.

For our numerical analyses, the following rescaling factors such as $V_{c 1}=x, V_{c 2}=y$ are used where $V_{c 1}$ and $V_{c 2}$ are the voltages across the capacitors $c_{1}$ and $c_{2}$, respectively. $G(y)$ is the nonlinear function of the diode. $f_{1}$ and $f_{2}$ are the amplitudes of the external periodic forces with the frequencies $\omega_{1}$ and $\omega_{2}$, respectively. The gain of the Wien bridge oscillator is controlled by $R_{i}$ and $R_{f}$ such that $k=$ $1+R_{f} / R_{i}$, which should be maintained above 3 so as to maintain the oscillations. Practically this can be written as $k=3+\Delta k$ where $\Delta k$ is a small parameter to initiate the oscillations. Hence, we have chosen $k=4$ for our system analysis.

\section{Results and Discussion of Experimental and Numerical Investigation}

3.1. Analyses Using Phase Diagram, Power Spectrum, and Maximal Lyapunov Exponent. The numerical analyses for the system associated equations are performed with the help of ode45 solver-MATLAB. The experimental and numerical phase trajectories (Figure 3 ) are accrued for the voltage across capacitor $c_{1}\left(x\right.$ axis) and sinusoidal force $f_{1} \sin \omega_{1} t$ ( $y$ axis) to scrutinize the emanating dynamics.

During the study of dynamics of the system, it is observed that when the external sinusoidal forcing signal $f_{1} \sin \omega_{1} t$ boosts the system, the aperiodic chaotic dynamics is prognosticated for chosen fixed parameters in a good frequency range, suitable for high frequency applications. When the 

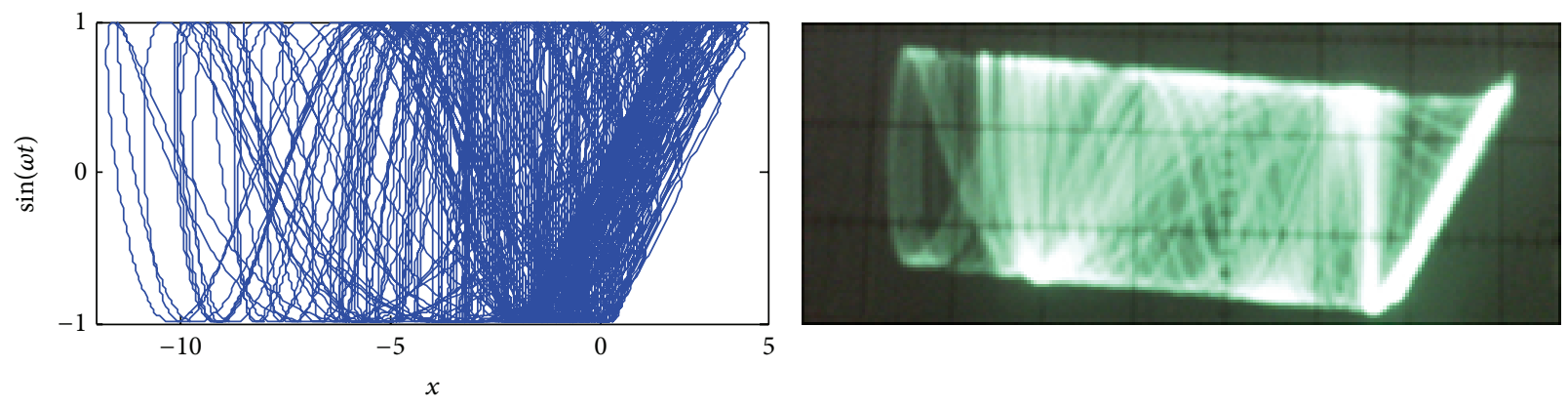

(a)
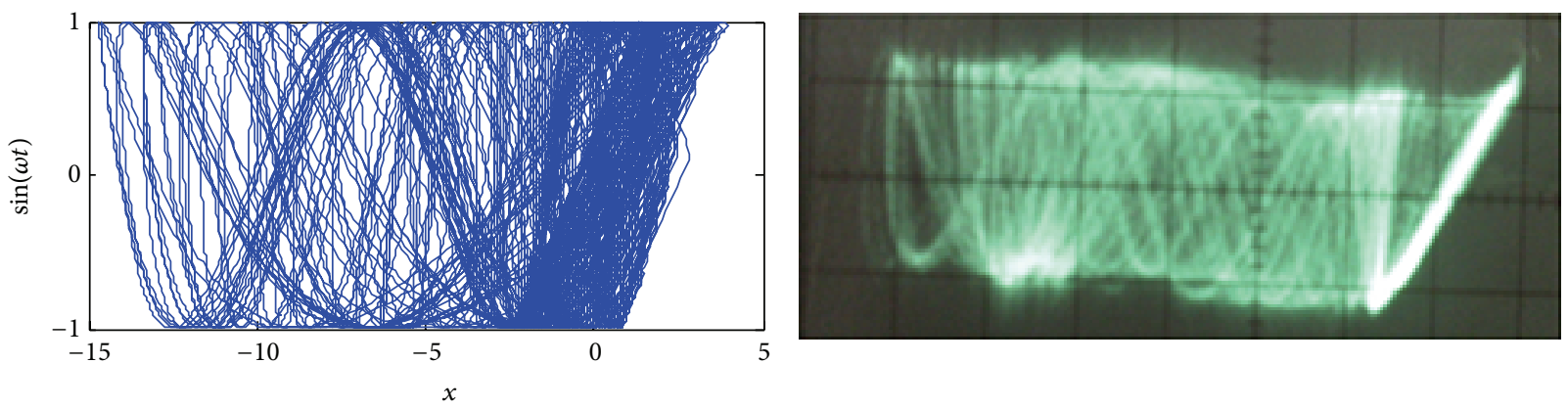

(b)
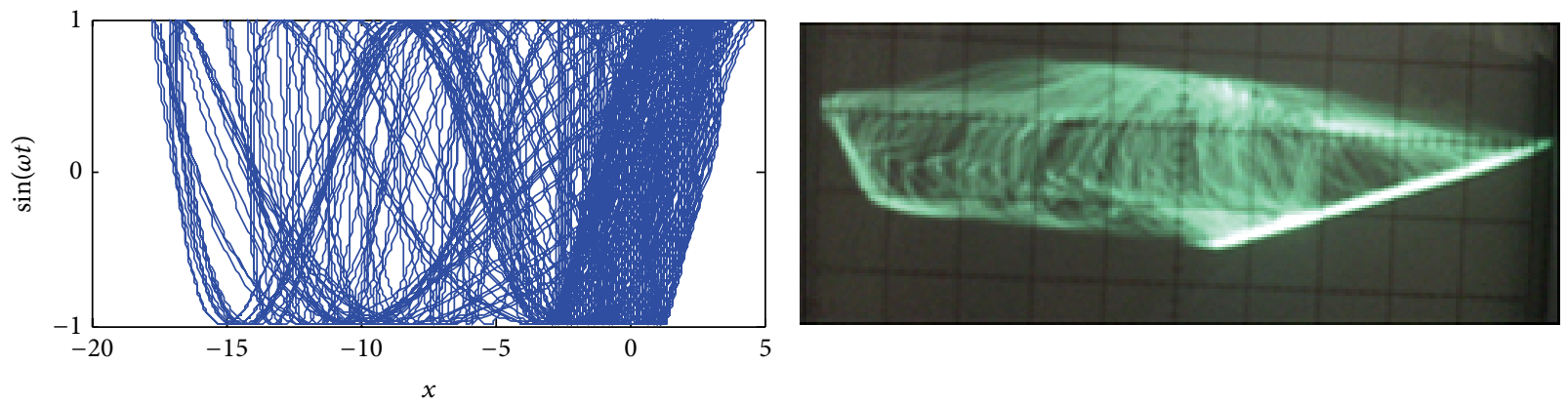

(c)

FiguRE 3: Numerical phase portrait (left); experimental phase portrait (right): (a) chaotic attractor at $f_{2}=0.1$, (b) strange nonchaotic attractor at $f_{2}=1.55$, and (c) quasiperiodic torus attractor at $f_{2}=2.0$.

dynamics of the system is set at the nonlinear regime by fixing appropriate experimental values discussed in [25] with the frequency $\omega_{1}=4.3$, nonlinear phenomena like quasiperiodic torus are observed at $f_{1}=0.5$, then chaotic attractor at $f_{1}=1.8$, followed by periodic windows and again chaotic attractor for the slight changes in the variable parameter.

Consequently, the study of the system is extended for further investigation with the additional external periodic force $f_{2} \sin \omega_{2} t$. While the dynamics of the system oscillate in the chaotic regime at $\omega_{1}=4.3$ and $f_{1}=1.8$, the amplitude $f_{2}$ of the second force is slowly increased with the frequency fixed at golden mean ratio $(\sqrt{5}+1) / 2$. During the variation of the amplitude $f_{2}$, the system continues to remain in the chaotic state (Figure 3(a)) for a range, $f_{2}=0$ to 1.54 , after which it slowly leaves the chaotic state and dynamics starts to exhibit SNAs from $f_{2}=1.55$ to $f_{2}<2.0$ (Figure 3(b)) whereas further variation in the amplitude $\left(f_{2} \geq 2\right)$ makes the system yield the dynamics of quasiperiodic torus attractors. The corresponding power spectra are shown in Figure 4.

Qualifying this, the maximal Lyapunov exponent (LE) is calculated to characterize the dynamics as the function of $f_{2}$ as [26]

$$
\lambda=\lim _{T \rightarrow \infty} \frac{1}{T} \sum_{t=0}^{T-1} \log \left(\frac{d\left(x_{0}, t\right)}{d\left(x_{0}, 0\right)}\right),
$$

where $d\left(x_{0}, t\right)$ is the measure of the distance between two trajectories at time $t$ and $d\left(x_{0}, 0\right)$ is that of the two initial trajectories.

The plot of the largest LE is shown in Figure 5. The calculation yields positive LE for aperiodic dynamics for the value of $f_{2}$ below 1.55 due to the exponential divergence of chaotic trajectories. For the range of $f_{2}$ from 1.55 , the system exhibits negative LE indicating quasiperiodic state such that 


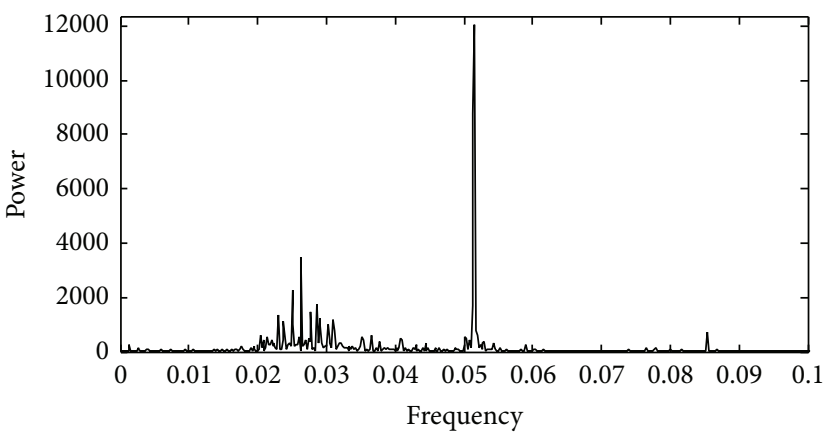

(a)

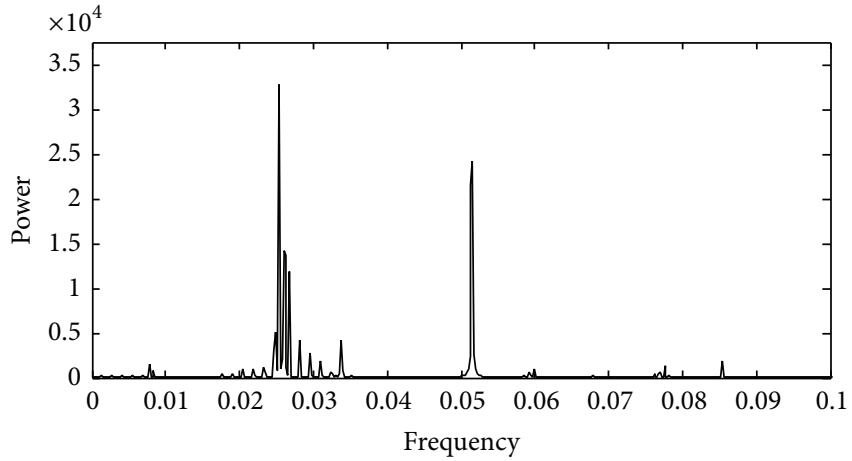

(b)

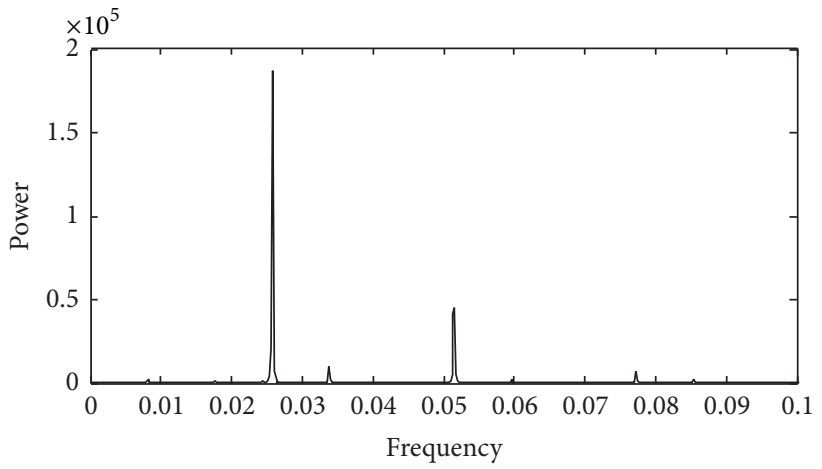

(c)

Figure 4: Power spectrum: (a) chaos at $f_{2}=0.1$, (b) SNA at $f_{2}=1.55$, and (c) torus at $f_{2}=2.0$.

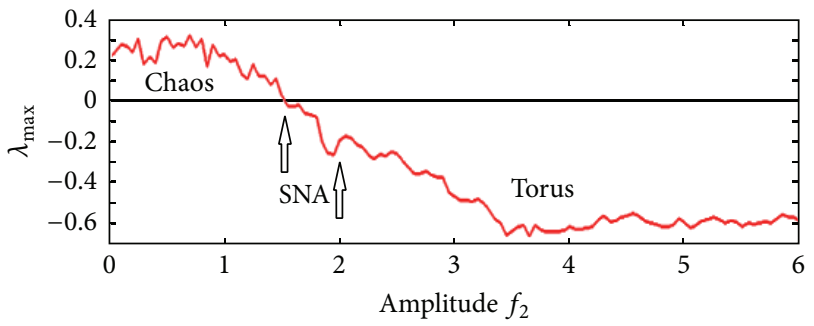

FIGURE 5: Variation of maximal Lyapunov exponent with the amplitude $f_{2}$.

SNA rises as an intermediate dynamics at $f_{2}=1.55$ followed by the torus at $f_{2} \geq 2.0$.

\subsection{Analysis of 0-1 Test and Projection of Poincaré Section} in $(\varphi-x)$ Plane. Characterizing the regular and irregular dynamics using the binary test, namely, "0-1 test" introduced by Gottwald and Melbourne [27] is a unique and useful method as it depicts the regular and complex dynamics and analyses the presence of SNAs during the transition between regular and chaotic dynamics $[11,27]$. The $0-1$ test uses only a time series data irrespective of the knowledge of dimension of underlying system. Consequently, this test results in and around unit value for chaotic dynamics, approaches zero for regular dynamics and lies between 0 and 1 for the regime of SNAs. Gottwald and Melbourne's 0-1 test implemented with modified mean square displacement [27] has proved to be one of the useful tools for our system analyses to distinguish SNA among the chaotic and quasiperiodic regime.

The analysis of $0-1$ test which is actually the method of computing the correlation coefficient $K_{c}$ is calculated as follows.

Let $N$ be the total length of time series data and let $\varphi(t)$ be an observable with $t=1,2,3, \ldots, N$. The definition of correlation coefficient is given as

$$
\begin{aligned}
K_{c} & =\operatorname{correlation}(\xi, \Delta) \\
& =\frac{\operatorname{covariance}(\xi, \Delta)}{\sqrt{\text { variance }(\xi) \text { variance }(\Delta)}} \in[-1,1],
\end{aligned}
$$

where $\xi$ denote the vectors such as $\left\{1,2, \ldots, n_{\text {cut }}\right\}$ and $\Delta$, the series of modified mean displacement (i.e.) $\Delta=$ $\left\{M_{m}(1), M_{m}(2), \ldots, M_{m}\left(n_{\text {cut }}\right)\right\}$ with $n_{\text {cut }} \ll N$.

Mean square displacement is determined using the translation variables $p(n)$ and $q(n)$ (function of $\varphi(t)$ ) by applying directly its definition [27] with $n_{\text {cut }} \ll N$. As in [27], the modification to the mean square displacement is necessary as it regularizes the linear behavior of its old version. Evidently, its modification permits a good prediction of the asymptotic growth rate $K_{c}$.

For our system studied, this test is carried out by considering the series of time dependent variables, $x(t)$. In order to enhance the performance of this test, it is recommended [11] to take the total data of length $N\left(5 \times 10^{5}\right)$ divided into 


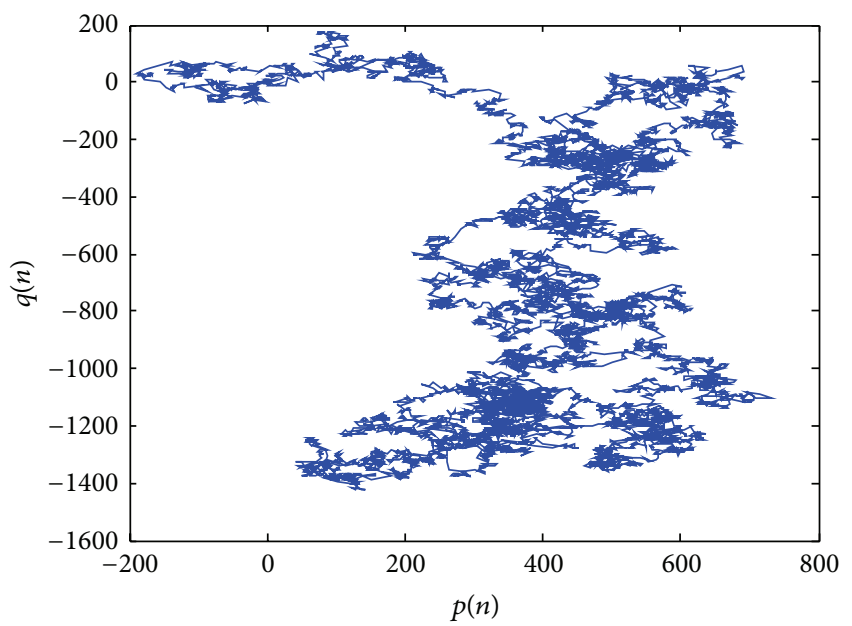

(a)

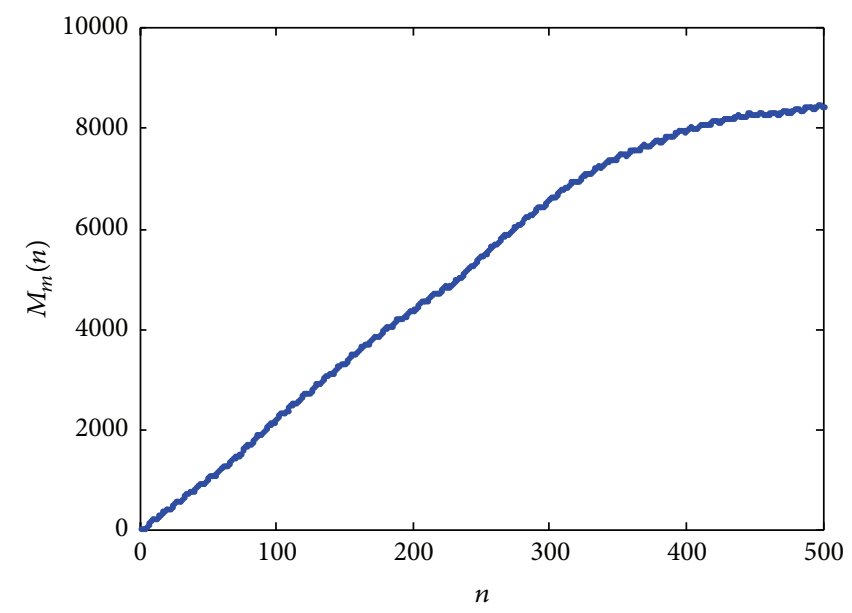

(b)

Figure 6: (a) Path of translation variables $(p(n)$ versus $q(n))$ and (b) plot of mean square displacement $M_{m}(n)$ for SNA dynamics at $f_{2}=1.55$.

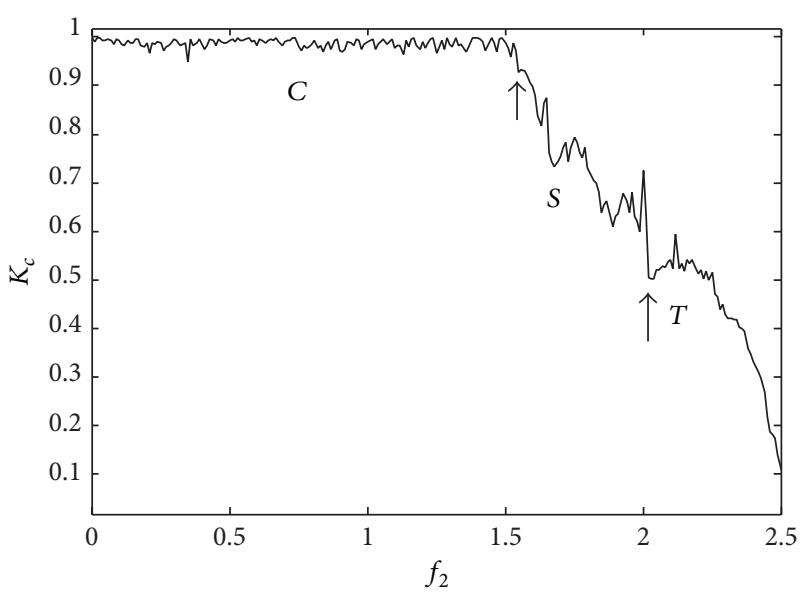

Figure 7: "0-1 test"-variation of the correlation $\left(K_{c}\right)$ with the amplitude $f_{2}$.

10 segments, each of segments having equal number of data. Then, the correlation coefficient $K_{c}$ is computed for each segment. Finally it is found by taking average over them and this method is repeated for each of the varying amplitude $f_{2}$ for a selected range.

The plot of translation variables which makes a fractal walk and modified mean square displacement which oscillates initially, later which increases linearly as the time passes on are shown in Figure 6 for the case of SNA and "0-1 test" in Figure 7.

It follows that in Figure 7 chaotic regime is indicated by the value $K_{c}$ oscillating near 1 . For a range of amplitude lying between $1.55 \leq f_{2}<2$, the transition to SNA takes birth in the route of merging of bands followed by the intermittency route and then blow out bifurcation route for which $K_{c}$ descends down that lies between 0 and 1 indicated by the arrow. The region of torus emerges for $f_{2} \geq 2$ indicated by second arrow. The range of SNA regime can be well defined from that of the chaotic and quasiperiodic dynamics.

The projection of Poincaré section in the $(\varphi-x)$ plane helps to understand the birth route of SNAs which is clearly shown in (Figure 8) for the band merging route, intermittency route, and blowout bifurcation route.

3.3. Spectral Analysis of Log-Log Plot and Autocorrelation Plot. A qualitative characterizing measure using the relation of partial Fourier transformation $X(\omega, T)=\sum_{n=1}^{T} x_{n} \exp (i 2 \pi n)$ reveals singular continuous spectrum for SNA [28], obeying the power law relation, $|X(\omega, T)|^{2} \sim T^{\beta}$ with $1<\beta<2$ and $\beta$ being scaling component or the slope of the fitted linear of the spectrum. Indeed the slope of the linear curve in the plot of $\log X|(\omega, T)|^{2}$ versus $\log T$ (Figure 9) lies at $\beta=1.6$ for SNAs that correspond to the nonchaotic motion with strange behavior, whereas in the case of chaos, the spectrum impart continuous broad band observance with $\beta$ more than 2 indicating irregular dynamics. The autocorrelation plot (Figure 10) of SNA diminishes after a finite interval of time proving the property of SNA.

\section{Conclusion}

In this paper, a modified nonautonomous model of standard Wien bridge oscillator is investigated to reveal the simplicity of the low dimensional system acting under the force of quasiperiodic signals using a simple diode as a nonlinear device. The system exhibits complex behavior belonging to chaotic attractors and SNAs. In the study, birth of SNAs takes place in three routes-merging of bands followed by intermittency route and blowout bifurcation route. Numerical approach, namely, "0-1 test" which has been newly introduced for characterizing SNA dynamics that occur during the transition between chaotic and regular dynamics is reported in the present work. Additional numerical analyses such as maximal Lyapunov exponent, power spectrum, Poincaré 


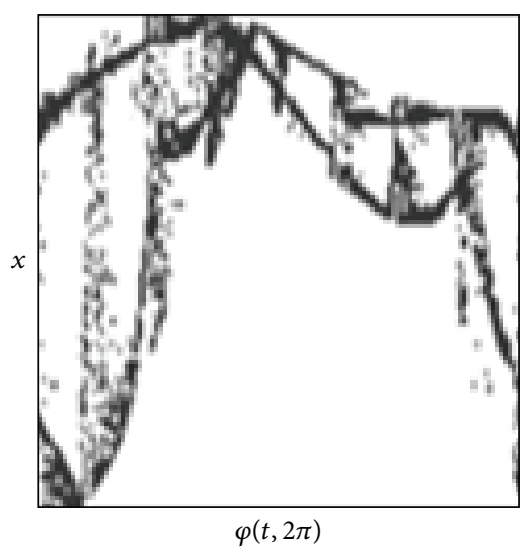

(a)

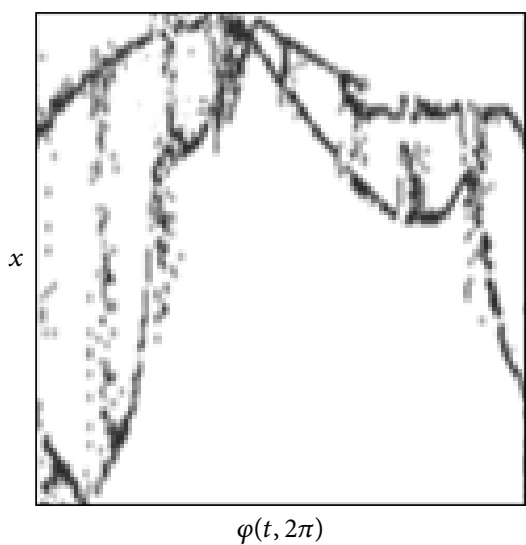

(b)

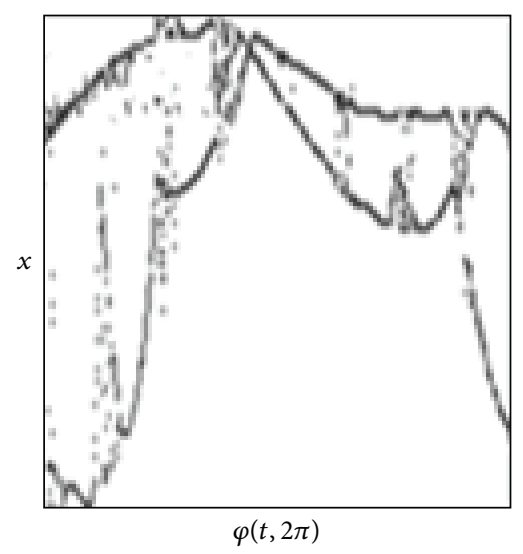

(c)

FIgURE 8: Projection of Poincaré section $\bmod (\varphi, 2 \pi)$ in $\varphi-x$ plane (a) birth of SNA in the band merging route (b) birth of SNA in the intermittency route and (c) birth of SNA in the blowout bifurcation route.

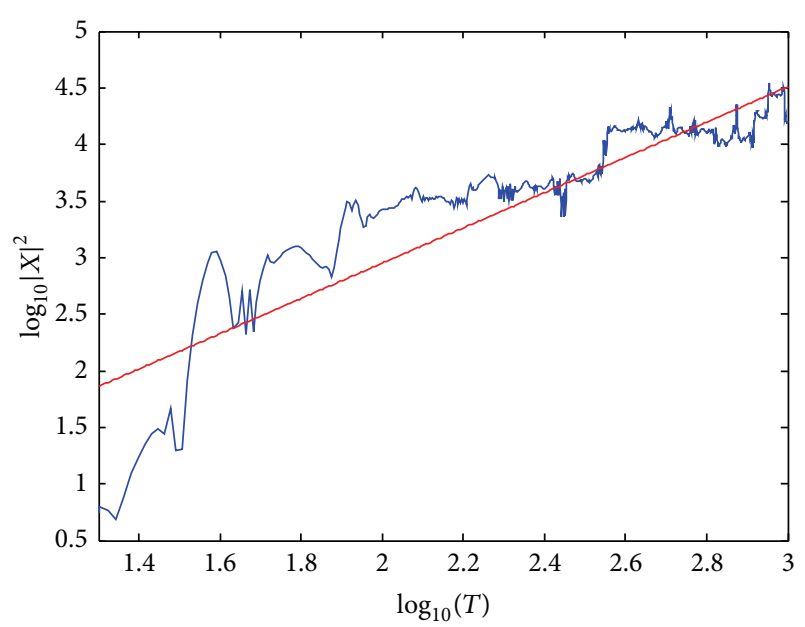

FIGURE 9: Singular continuous spectrum (SNA dynamics) with slope $\beta=1.6$ at $f_{2}=1.55$.

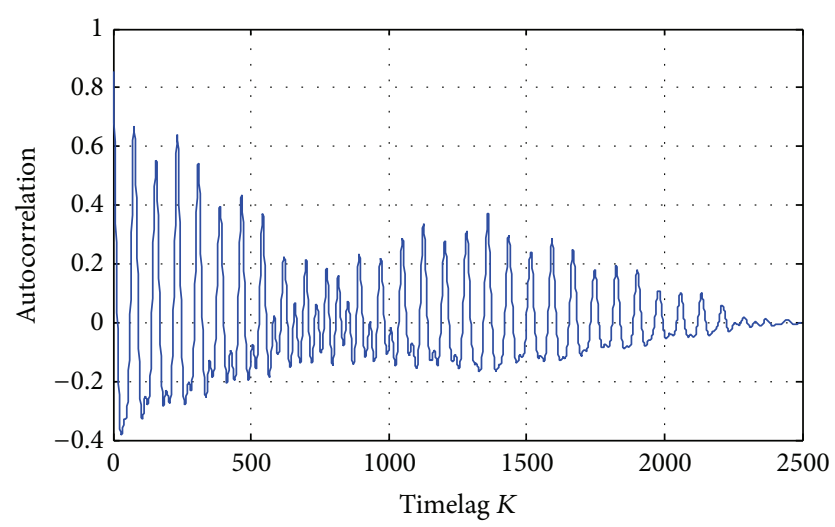

FIgURE 10: Autocorrelation function of SNA at $f_{2}=1.55$.

projection, spectral analysis of log-log plot, autocorrelation are carried out supporting the experimental results. We also add that SNAs can meet the applications replacing chaotic attractors. Chaotic attractors are observed for a wide range of variable parameter whereas SNAs reveal their presence only for a short range of system variant [21]. Such signals can be used for the secure transmission of digital signals through the synchronization between two quasiperiodically forced nonlinear systems, provided the frequencies ratio remain irrational and hence the phases of the quasiperiodic driving can be matched. The synchronization property of SNA along with their short range existence may find essential application [21-24] in secure communication processes.

\section{Conflict of Interests}

The authors declare that there is no conflict of interests regarding the publication of this paper.

\section{References}

[1] C. Grebogi, E. Ott, S. Pelikan, and J. A. Yorke, "Strange attractors that are not chaotic," Physica D: Nonlinear Phenomena, vol. 13, no. 1-2, pp. 261-268, 1984.

[2] A. S. Pikovsky and U. Feudel, "Correlations and spectra of strange non-chaotic attractors," Journal of Physics A: Mathematical and General, vol. 27, no. 15, pp. 5209-5219, 1994.

[3] A. Venkatesan, K. Murali, and M. Lakshmanan, "Birth of strange nonchaotic attractors through type III intermittency," Physics Letters A, vol. 259, no. 3-4, pp. 246-253, 1999.

[4] P. Awadhesh, M. Agrawal, and R. Ramaswamy, Strange Nonchaotic Attractors in Driven Delay-Dynamics, Nonlinear Dynamics Narosa Publishing House, 2009.

[5] F. J. Romeiras and E. Ott, "Strange nonchaotic attractors of the damped pendulum with quasiperiodic forcing," Physical Review A, vol. 35, no. 10, pp. 4404-4413, 1987.

[6] A. Bondeson, E. Ott, and T. M. Antonsen, "Quasiperiodically forced damped pendula and Schrödinger equations with quasiperiodic potentials: implications of their equivalence," Physical Review Letters, vol. 55, no. 20, pp. 2103-2106, 1985.

[7] M. Ding and J. A. Scott Relso, "Phase-Resetting map and the dynamics of quasi-periodically forced biological oscillators," International Journal of Bifurcation and Chaos, vol. 4, no. 3, pp. 553-567, 1994. 
[8] A. Venkatesan, M. Lakshmanan, A. Prasad, and R. Ramaswamy, "Intermittency transitions to strange nonchaotic attractors in a quasiperiodically driven Duffing oscillator," Physical Review EStatistical Physics, Plasmas, Fluids, and Related Interdisciplinary Topics, vol. 61, no. 4, pp. 3641-3651, 2000.

[9] A. Venkatesan and M. Lakshmanan, "Different routes to chaos via strange nonchaotic attractors in a quasiperiodically forced system," Physical Review E, vol. 58, no. 3, pp. 3008-3016, 1998.

[10] K. Suresh, A. Prasad, and K. Thamilmaran, "Birth of strange nonchaotic attractors through formation and merging of bubbles in a quasiperiodically forced Chua's oscillator," Physics Letters A, vol. 377, no. 8, pp. 612-621, 2013.

[11] R. Gopal, A. Venkatesan, and M. Lakshmanan, "Applicability of 0-1 test for strange nonchaotic attractors," Chaos, vol. 23, no. 2, Article ID 023123, 2013.

[12] W. L. Ditto, M. L. Spano, H. T. Savage, S. N. Rauseo, J. Heagy, and E. Ott, "Experimental observation of a strange nonchaotic attractor," Physical Review Letters, vol. 65, no. 5, pp. 533-536, 1990.

[13] T. Zhou, F. Moss, and A. Bulsara, "Observation of a strange nonchaotic attractor in a multistable potential," Physical Review A, vol. 45, no. 8, pp. 5394-5400, 1992.

[14] W. X. Ding, H. Deutsch, A. Dinklage, and C. Wilke, "Observation of a strange nonchaotic attractor in a neon glow discharge," Physical Review E: Statistical Physics, Plasmas, Fluids, and Related Interdisciplinary Topics, vol. 55, no. 3, pp. 3769-3772, 1997.

[15] A. Prasad, R. Ramaswamy, I. I. Satija, and N. Shah, "Collision and symmetry breaking in the transition to strange nonchaotic attractors," Physical Review Letters, vol. 83, no. 22, pp. 45304533, 1999.

[16] J. J. Stagliano Jr., J.-M. Wersinger, and E. E. Slaminka, "Doubling bifurcations of destroyed $\mathbb{T}^{2}$ tori," Physica D: Nonlinear Phenomena, vol. 92, no. 3-4, pp. 164-177, 1996.

[17] J. F. Heagy and S. M. Hammel, "The birth of strange nonchaotic attractors," Physica D, vol. 70, no. 1-2, pp. 140-153, 1994.

[18] T. Nishikawa and K. Kaneko, "Fractalization of a torus as a strange nonchaotic attractor," Physical Review E: Statistical Physics, Plasmas, Fluids, and Related Interdisciplinary Topics, vol. 54, no. 6, pp. 6114-6124, 1996.

[19] T. Yalçinkaya and Y.-C. Lai, "Blowout bifurcation route to strange nonchaotic attractors," Physical Review Letters, vol. 77, no. 25, pp. 5039-5042, 1996.

[20] A. Prasad, V. Mehra, and R. Ramaswamy, "Strange nonchaotic attractors in the quasiperiodically forced logistic map," Physical Review E: Atomic, Molecular, and Optical Physics, vol. 57, no. 2, pp. 1576-1584, 1998.

[21] R. Ramaswamy, "Synchronization of strange nonchaotic attractors," Physical Review E: Statistical, Nonlinear, and Soft Matter Physics, vol. 56, no. 6, pp. 7294-7296, 1997.

[22] C.-S. Zhou and T.-L. Chen, "Robust communication via synchronization between nonchaotic strange attractors," Europhysics Letters, vol. 38, no. 4, pp. 261-265, 1997.

[23] A. Prasad, S. S. Negi, and R. Ramaswamy, "Strange nonchaotic attractors," International Journal of Bifurcation and Chaos in Applied Sciences and Engineering, vol. 11, no. 2, pp. 291-309, 2001.

[24] K. Thamilmaran, D. V. Senthilkumar, A. Venkatesan, and M. Lakshmanan, "Experimental realization of strange nonchaotic attractors in a quasiperiodically forced electronic circuit," Physical Review E: Statistical, Nonlinear, and Soft Matter Physics, vol. 74, no. 3, Article ID 036205, 2006.
[25] R. Rizwana, I. R. Mohamed, K. Srinivasan, and M. Inbavalli, "Simple nonautonomous Wien-bridge oscillator based chaotic circuit," in Proceedings of the 2nd International Conference on Devices, Circuits and Systems (ICDCS '14), pp. 1-4, IEEE, Coimbatore, India, March 2014.

[26] M. Lakshmanan and S. Rajasekar, Nonlinear Dynamics: Integrability, Chaos and Patterns, Advanced Texts in Physics, Springer, Heidelberg, Germany, 2003.

[27] G. A. Gottwald and I. Melbourne, "On the implementation of the 0-1 test for chaos," SIAM Journal on Applied Dynamical Systems, vol. 8, no. 1, pp. 129-145, 2009.

[28] A. Witt, U. Feudel, and A. Pikovsky, "Birth of strange nonchaotic attractors due to interior crisis," Physica D: Nonlinear Phenomena, vol. 109, no. 1-2, pp. 180-190, 1997. 

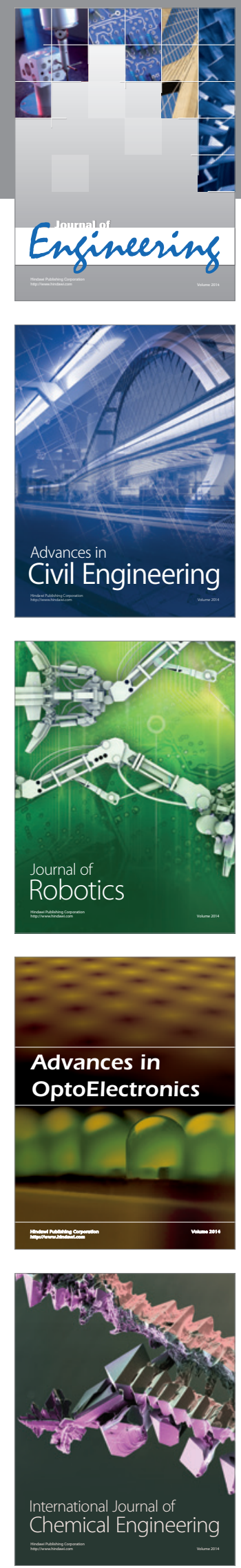

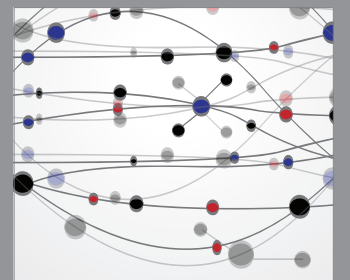

The Scientific World Journal
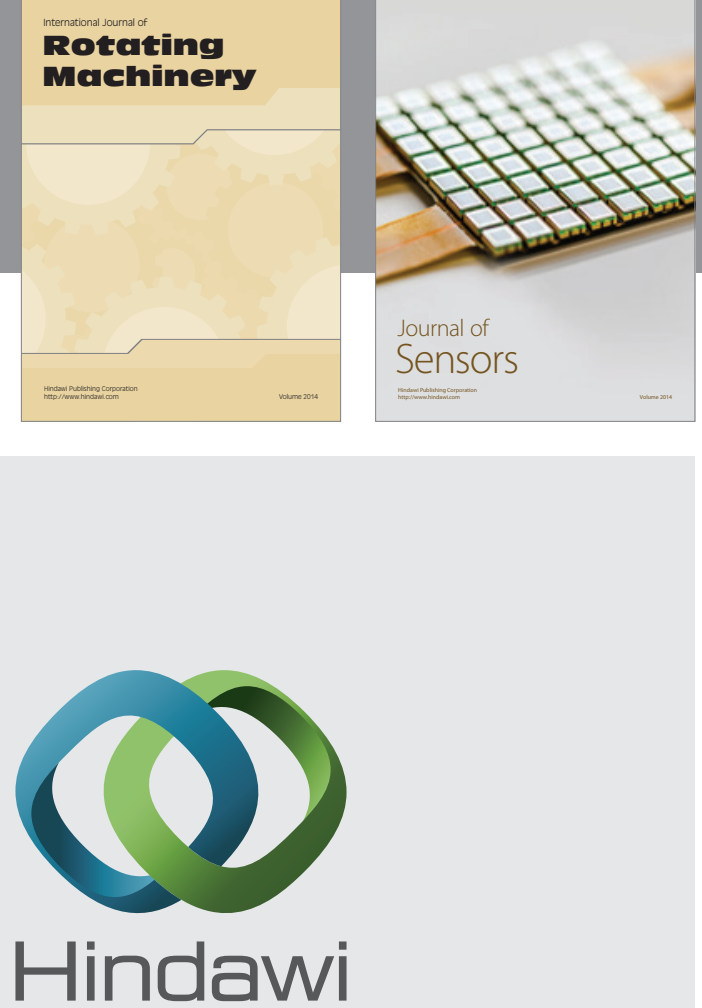

Submit your manuscripts at http://www.hindawi.com
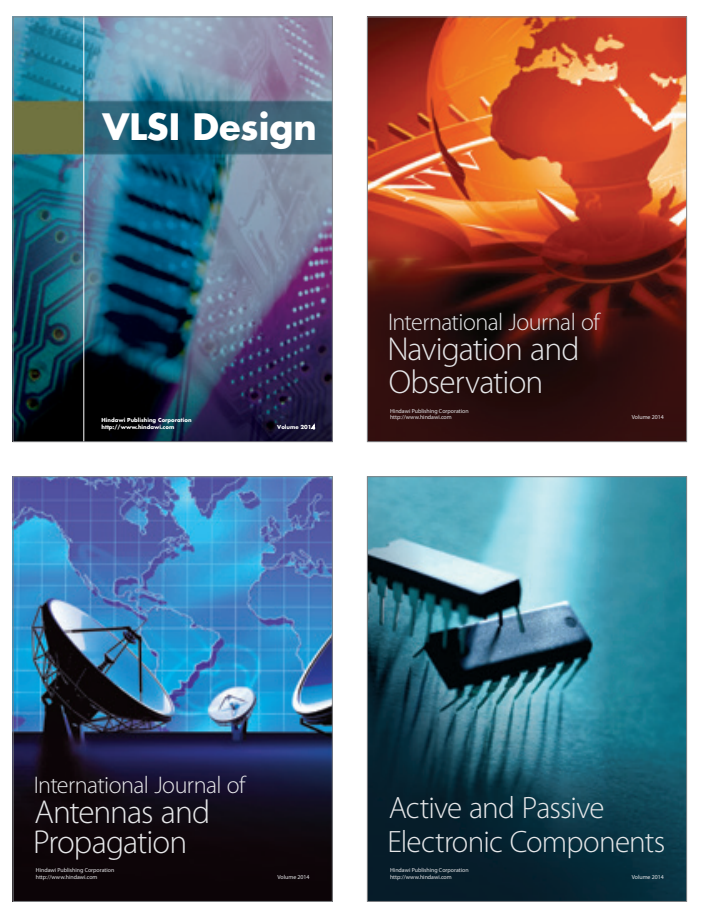
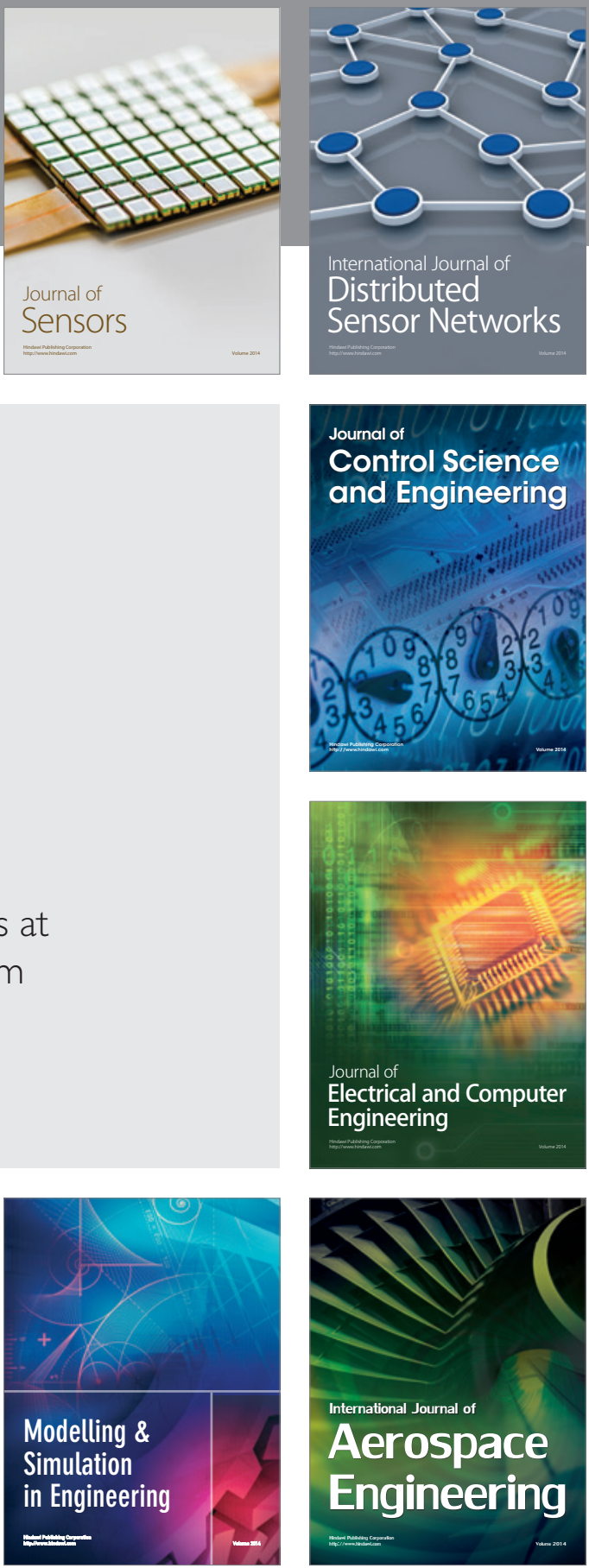

Journal of

Control Science

and Engineering
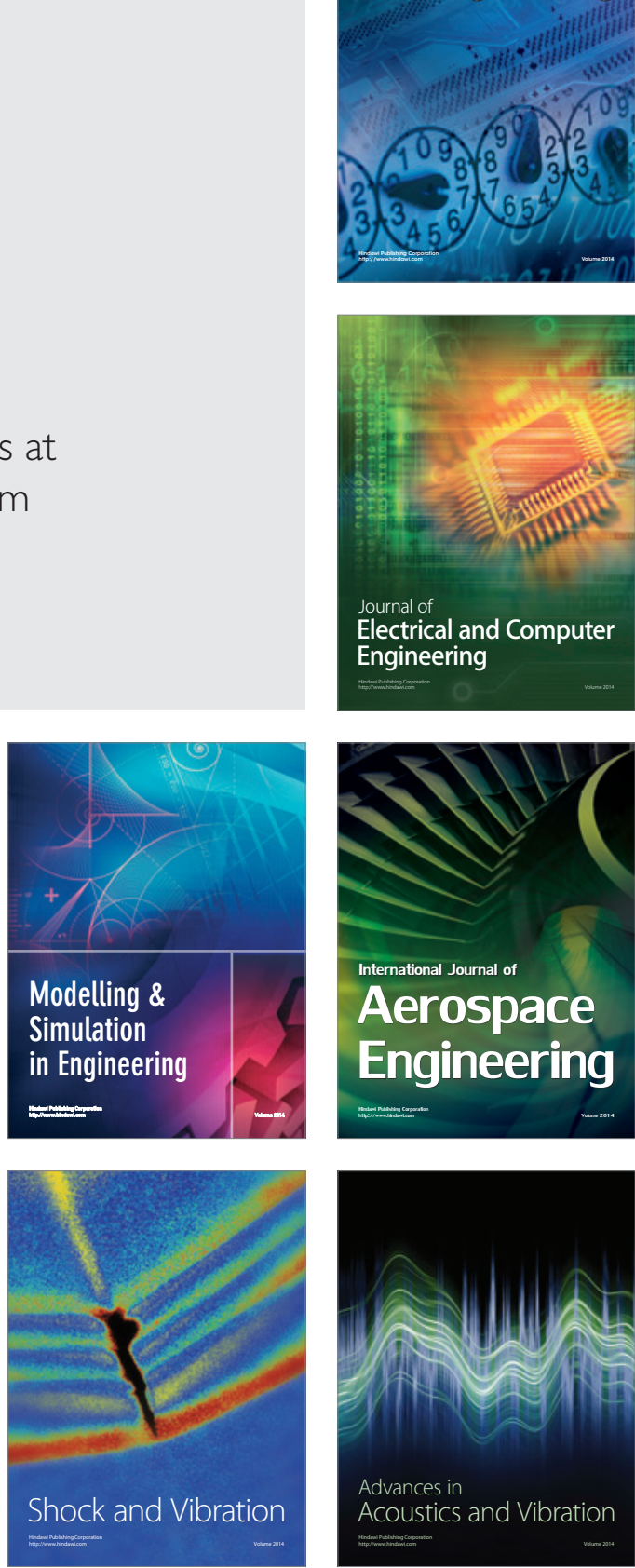